\title{
Adaptive Fuzzy Sliding Mode Optimum Control, Using the Fuzzy Control in Flat Robot
}

\author{
Jafar Shabani \\ Student of master education in electricity control, Dar-Al Fonoun Nonprofit University, Qazvin \\ Shabanijafar6@gmail.com
}

\begin{abstract}
Control of an industrial robot consist of nonlinear terms that causes the uncertainty and external turmoil and must be considered in control law designing. In this study, a control strategy will be presented for the robot arms, based on fuzzy logic control connection and sliding mode control (SMC). The using motivation of SMC in robotic is mainly the admirable features of it such as design simplicity and resistivity. However, the chattering effect could be destructive especially in typical SMC. In this article, the problem have been resolved, adapting between a Comparative Fuzzy Sliding Mode Control to a sliding flat (PID). For the suggested method, a fuzzy logic control has been used for the providing of impact control signals. In addition, the output gain of the Fuzzy Sliding Mode Control (FSMC), is set by a supervised fuzzy system as online to prevent of chattering. System stability is guaranteed from the aspect of Lyapunov stability theorem. The numerical simulation, using the dynamic model of a rigid flat robot arm with three degree of freedom, comparing the results of this simulation with general SMCs with PID sliding level, reveals the efficiency of robot system control. The suggested AFSMC can fulfilled the route tracking of intended path and is resistant against of uncertainties and disturbances.
\end{abstract}

Keywords: Fuzzy control, Robot, Sliding mode control, Uncertainty

\section{Introduction}

In control science we are faced to controller designing problem for a system so the system be able to do a considered operation. Due to systems diversity and of course various control targets, the researchers have been developed an extend range of control procedures. Sliding Mode Control (SMC) from 1970 until now has been concerned greatly due to its stability against the parameter changes and confusions. Since the rigid robots have been introduced as the under control systems, the different and various procedures have been suggested and tested, controlling them. This operation is difficult because of the nonlinear terms and the effects of type coupling in robotic systems (capisani et al, 2007). Different methods such as feedback linearization, model prediction control and sliding mode control have been followed, solving this problem. In robotic field, the feedback linearization is done by the inverse dynamic control. The control of calculated torque has been developed based on feedback linearization (chang and chen, 2000). Comparative control methods can be applied, facing to unknown robot dynamics. In these methods the linear parameterization should be considered, means that unknown parameters should be linear, constant or change slowly. However if the dynamic robotic systems be nonlinear, transient with high coupling the linear parameterization may not be applicable (Ho et al,2007). Sliding Mode Control is one of the most effective methods in nonlinear stable control that provides the stable system dynamics when they have been controlled in sliding mode. The first step in SMC designing is the sliding surface selection to model the acceptable application of closed loop in fluctuated space state (Zhu et al, 2010). Second step is the designing of an impact control rule that system state paths move towards the sliding surface and remain on it. The system path in time range before the reaching on sliding surface called Reaching Phase. When the system path reaches to the sliding surface, remains on it and slides along it to reaches to origin. System path sliding through the sliding surface to the origin is called sliding mode. Under the given circumstances the SMC is stable against the system disorders and outer confusions (Vakula, Sudha, 2012). In practice the switching frequency limitation causes that system situations don't remain on switching surface and oscillate around that. These oscillations called chattering that is unacceptable. In this article a novel control algorithm has been developed using the fuzzy method and Proportional-Integral-Derivative sliding surface conjugation.

Suggested method combined the comparative fuzzy algorithm with stable control technique for the guarantee of unknown robotic system stable detection efficiency. The suggested algorithm performed via the simulation of flat robot arm with 3 degree freedom. The suggested method has been compared with popular sliding mode controllers for the robot arms in control application and benefit sides. This article consists of below sections: section 1 presents the dynamic model properties of a robot, section 2 introduced the properties of fuzzy PID controller, section 3 presents a fuzzy sliding mode control (FSMC) method, section 4 has been 
introduced the Adaptive Fuzzy Sliding Mode Control (AFSMC), section 5 stated the simulation results and section 6 summarized the results of the study.

\section{Description of Robot Arm Model}

The dynamic and robot control principles have been recognized as well. Here we use the dynamics in control algorithm and inference (Spong and Vidyasagar, 1989), therefore we have for the n-link robot with Euler- Lagrange equations:

$$
B(q) \ddot{q}+C(q, \dot{q}) \dot{q}+F_{d} \dot{q}+F_{s}(\dot{q})+\tau_{d}(q, \dot{q})+g(q)=\tau
$$

Where $q, \dot{q}, \ddot{q} \in R^{n}$ are joint location, velocity and acceleration vectors. $B(q)$ Shows the exact bounded positive inertia matrix, $C(q, \dot{q})$ Coriolis centripetal matrix, $g(q)$ gravity vector, $F_{d} \in R^{n \times n}$ and $F_{s}(\dot{q})$ respectively are the matrix of dynamic friction coefficient and static friction vector. $\tau_{d}(q, \dot{q})$ Confusion vector and not modeled dynamics and $\tau$ is the control vector that shows the applied torque on joints. The equation 1 could be written as below:

$$
\ddot{q}=-B^{-1}(q) C(q, \dot{q}) \dot{q}-B^{-1}(q) g(q)-d(t)+B^{-1}(q) \tau
$$

Where $d(t)=B(q)^{-1}\left(\left(F_{d} \dot{q}+F_{s} \dot{q}\right)+\tau_{d}(q, \dot{q})\right)$ shows the outer load confusion, nonlinear friction and not modelled dynamics. From the equation 2 dynamic equations could be written as below for the $n$-link robot.

$\ddot{q}=-D \dot{q}-E g(q)-d(t)+F u(t)$

Where $D=B^{-1}(q) C(q, \dot{q}), E=F=B^{-1}(q)$ and $u(t)=\tau$ are control vector. If there was uncertainty that showed parameter changes, it will be assumed that $\Delta \mathrm{D}, \Delta \mathrm{F}$ and $\Delta \mathrm{E}$ exist in system and the equation 3 will be rewritten as below:

$\ddot{q}=-(D+\Delta D) \dot{q}-(E+\Delta E) g(q)-d(t)+(F+\Delta F) u(t)$

Where the uncertainty is bounded as $\Delta D_{l} \leq|\Delta D| \leq \Delta D_{h}, \Delta E_{l} \leq|\Delta E| \leq \Delta E_{h}$ and $\Delta F_{l} \leq|\Delta F| \leq \Delta F_{h}$, the 1 and $\mathrm{h}$ subscribers show the upper and lower uncertainty.

\section{Fuzzy PID controller}

PID controller is used generally in industrial processes control, considering the simple structure and stable performance. Transfer function of a PID controller is as below (Eker, 2006):

$\mathrm{G}(\mathrm{S})=\mathrm{K}_{\mathrm{P}}+\mathrm{K}_{\mathrm{i}} / \mathrm{s}+\mathrm{K}_{\mathrm{d}} \mathrm{S}$

Where $\mathrm{K}_{\mathrm{p}}, \mathrm{K}_{\mathrm{i}}$ and $\mathrm{K}_{\mathrm{d}}$ are called Proportional, Integral and Derivational benefits respectively.

Other equivalent structure of PID controller is as below:

$\mathrm{U}(\mathrm{t})=\mathrm{K}_{\mathrm{P}}\left[\mathrm{e}(\mathrm{t})+1 / \mathrm{T}_{\mathrm{i}} \grave{o}^{\mathrm{t}} \mathrm{e}(\mathrm{t}) \mathrm{dt}+\mathrm{T}_{\mathrm{d}} \mathrm{e}(\mathrm{t})\right]$

Where $T_{i}=K_{P} / K_{i}$ and $T_{d}=K_{d} / K_{p}$ are called the time constant derivation and integral respectively. The situation of PID controller depends on suitable selection of PID benefits.

\section{Fig1. Fuzzy system as the PID controller indicator parameters}

\section{Fuzzy sliding mode control}

Sliding control is a stable control method and can be used, having the model of uncertainties and confusion parameters. A comparison between the sliding control and fuzzy control reveals that both method are similar in performance.

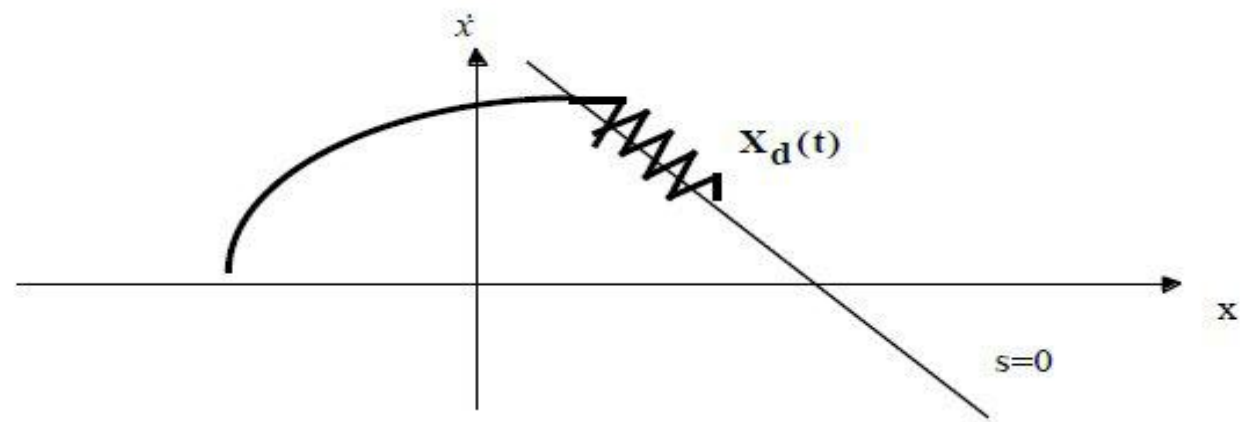

Fig2. Movement basic of sliding mode 
Fuzzy control has been replaced in most of current technologies. However the high amount of fuzzy rules complicates the analysis for the high rank systems. Therefore the more consideration has been occurred about the fuzzy sliding mode control (Yau and Chen, 2006). In this article a fuzzy interfere has been suggested for the achieving phase and fuzzy sliding mode control, elimination of chattering problem. The main benefit of this method is guaranteed the stable behavior of system. Second benefit of suggested method is that chattering elimination application is higher than the other similar methods.

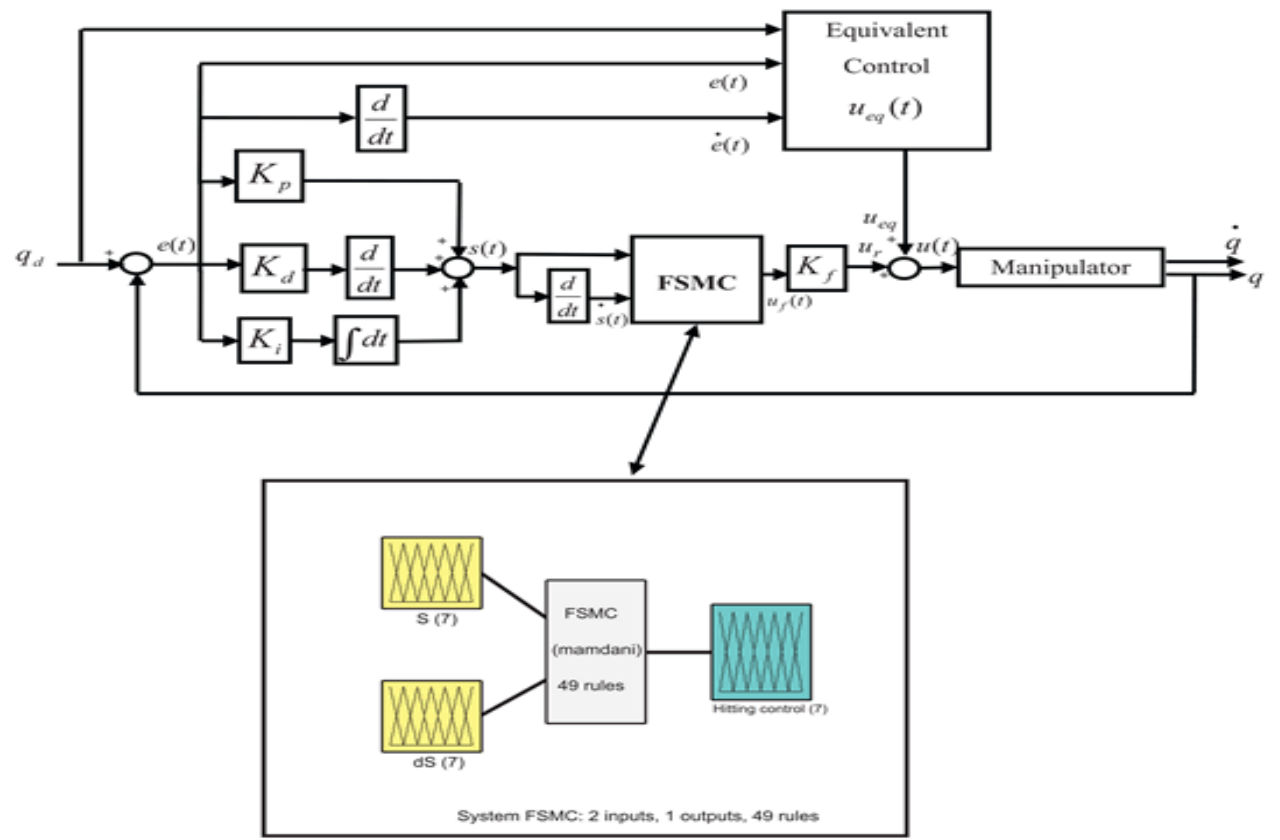

Fig3. Block diagram of fuzzy sliding mode control with PID sliding surface.

Achieving rule is considered as below:

$u_{r}(t)=K_{f} u_{f}(t)$

Where $\mathrm{K}_{\mathrm{f}}$ is the output normalization factor, $\mathrm{u}_{\mathrm{f}}(\mathrm{t})$ is the output of FSMC that is calculated by the normalized $\mathrm{s}$ (t) and $s(t)$. Fuzzy control rules can be written as a term of $\mathrm{s}(\mathrm{t})$ and $s(t)$ input linguistic variables to the $u_{f}(t)$ output linguistic variables can be shown as below:

$u_{f}(t)=F S M C(s(t), \dot{s}(t))$

Where $F S M C(s(t), \dot{s}(t))$ the functional characteristics of fuzzy language are decide diagrams. The $s(\mathrm{t})$ and $s(t)$ input linguistic variables and the $u_{f}(t)$ output linguistic variables have been shown in figures 3 and 4 respectively.

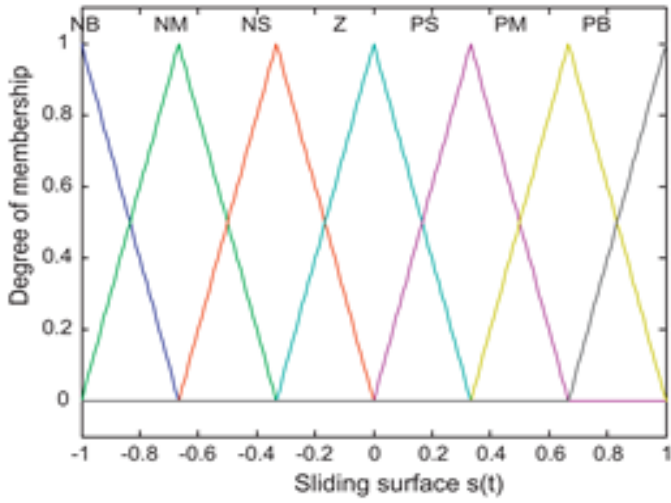

(a) $s(t)$

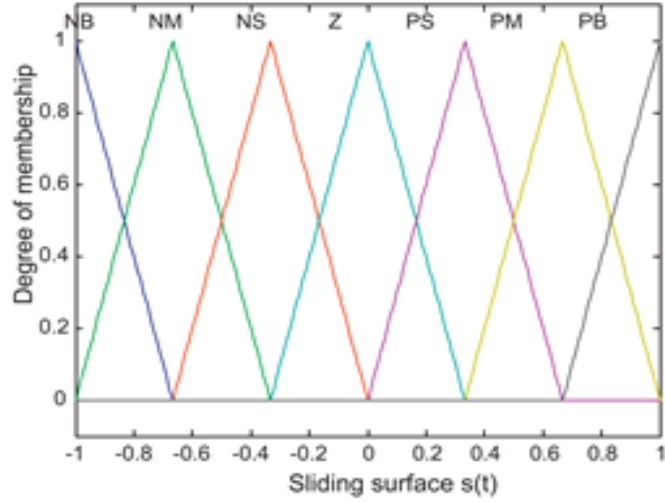

(b) $\dot{s}(t)$

Fig4. Sliding surface fuzzy set $\mathbf{s}(t)$ and sliding surface derivation $\dot{s}(t)$ 
These functions are divided to 7 fuzzy section contain NB (big negative), NM (middle negative), NS (small negative), Z (zero), PS (small positive), PM (middle positive) and PB (big Positive). The output fuzzy surface control has been shown in figure 5. The fuzzy linguistic rules are defined as below:

$$
R^{(l)}:: I F s(t) \text { is } A_{1}^{l} \text { and } \dot{s}(t) \text { is } A_{2}^{l} \text { THEN } u_{f}(t) \text { is } B^{l}
$$

Where $A_{1}^{l}$ and $A_{2}^{l}$ are the labels of input fuzzy sets. And also $B^{l}$ is the label of output fuzzy sets, while l= 1 , $2, \ldots, \mathrm{m}$ shows the number of if-then fuzzy rules.

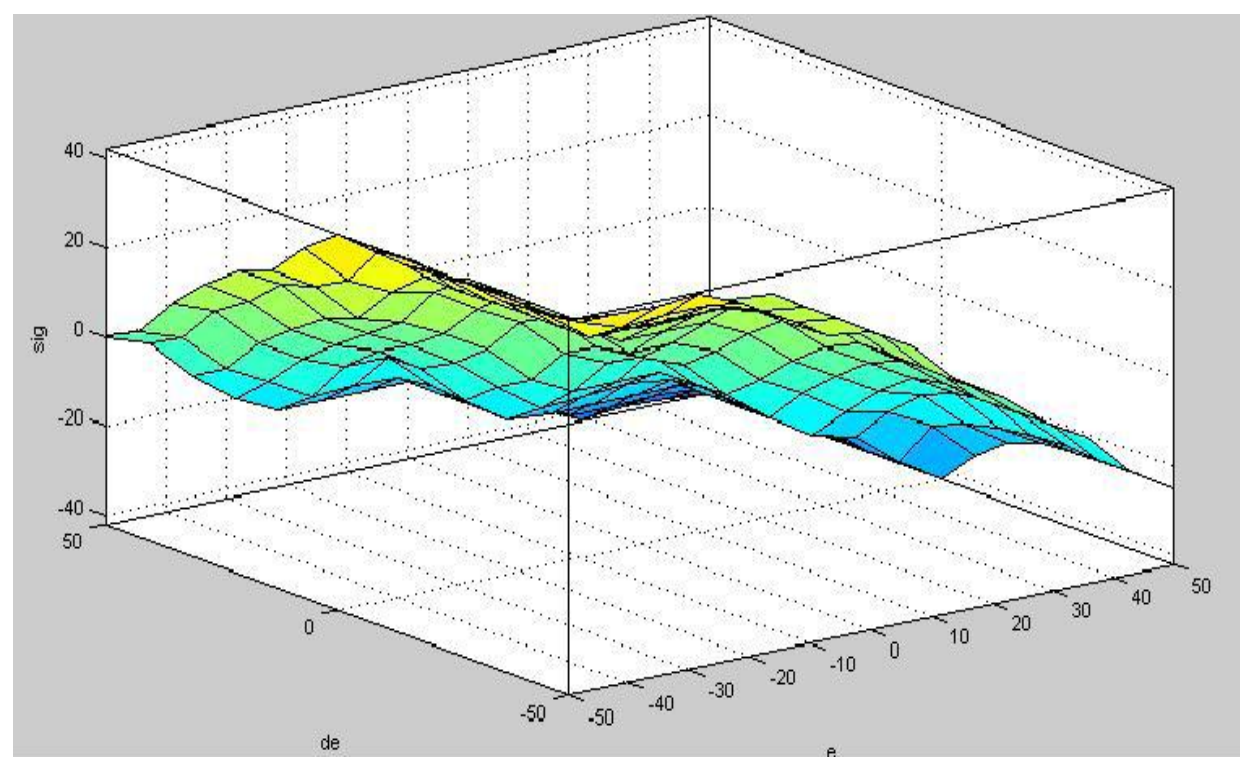

Fig5. $\mathbf{U}_{\mathrm{f}}(\mathrm{t})$ surface control

Table1. FSMC rule matrix

\begin{tabular}{llllllll}
\hline$u_{f}(t)$ & $s(t)$ & \multicolumn{7}{c}{} & & & \\
\cline { 2 - 7 }$\dot{s}(t)$ & NB & NM & NS & Z & PS & PM & PB \\
\hline NB & NB & NB & NB & NB & NM & NS & Z \\
NM & NB & NB & NB & NM & NS & Z & PS \\
NS & NB & NB & NM & NS & Z & PS & PM \\
Z & NB & NM & NS & Z & PS & PM & PB \\
PS & NM & NS & Z & PS & PM & PB & PB \\
PM & NS & Z & PS & PM & PB & PB & PB \\
PB & Z & PS & PM & PB & PB & PB & PB \\
\hline
\end{tabular}

\section{Adaptive fuzzy sliding mode controller}

Fuzzy controller, are the nonlinear controllers with an especial structure that are divided in two categories: non-adaptive fuzzy control and adaptive fuzzy control. The main target of adaptive control is the keeping steady of a system efficiency in absences of uncertainties. 


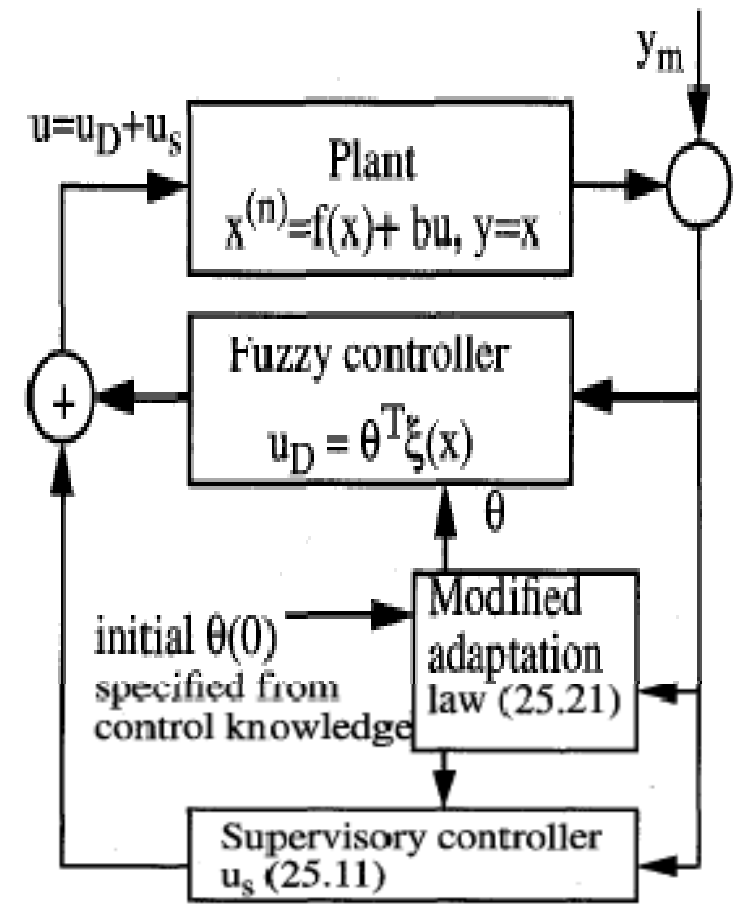

Fig6. Adaptive, direct and advanced control system with monitoring controller and changed adaptive base

In this section a fuzzy sliding mode control has been presented with a fluctuated control gain. The general form of suggested controller is shown in figure 7. A supervised interfere fuzzy system has been used for the adaptive control gain $\mathrm{K}_{\mathrm{f}}$. Here the supervised fuzzy control system has been written by the e error and its derivative as the initial assumption and $K_{f}=\operatorname{diag}\left\{k_{f 1}, k_{f 2}, \ldots . ., k_{f n}\right\}$ as the results of each rule. As it can be seen in figures 8 to 10 , the triangular membership functions with $50 \%$ coverage have been assumed for the input and output fuzzy variables. This level has been utilized for the adaptive setting of $K_{f}=\operatorname{diag}\left\{k_{f 1}, k_{f 2}, \ldots . ., k_{f n}\right\}$ as online form. The physical range of $(e, \dot{e})$ inputs is $\{-0.01,0.01\}$ and $K_{f}=\operatorname{diag}\left\{k_{f 1}, k_{f 2}, \ldots . ., k_{f n}\right\}$ output with 3 degree freedom is $\{4000,7500)$, which have been selected upon to trial and error. Fuzzy variables have been chosen as below:

$(e, \dot{e})=\{N B($ Negative Big ), NM (Negative Medium), NS (Negative Small ),

Z (Zero), PS (Positive Small ), PM (Positive Medium), PB (Positive Big)\};

$\left(K_{f}\right)=\{V V S($ Very Very Small $), V S$ (Very Small ),S (Small ), M (Medium)

\section{$B($ Big $), V B($ Very Big ),VVB (Very Very Big) $\}$}

A typical fuzzy control rule from the supervised type is stated as below:

$R^{(i)}:: I F e(t)$ is $E_{1}^{i}$ and $\dot{e}(t)$ is $E_{2}^{i} T H E N K_{f}$ is $G^{i}$

Where $E_{1}^{i}$ and $E_{2}^{i}$ are labels of input fuzzy sets and $G^{i}$ is the label of output fuzzy sets. While $\mathrm{i}=1,2, \ldots, \mathrm{p}$ is the number of if-then fuzzy rules. The fuzzy linguistic rules of supervised fuzzy system has been shown in figure 2 . 


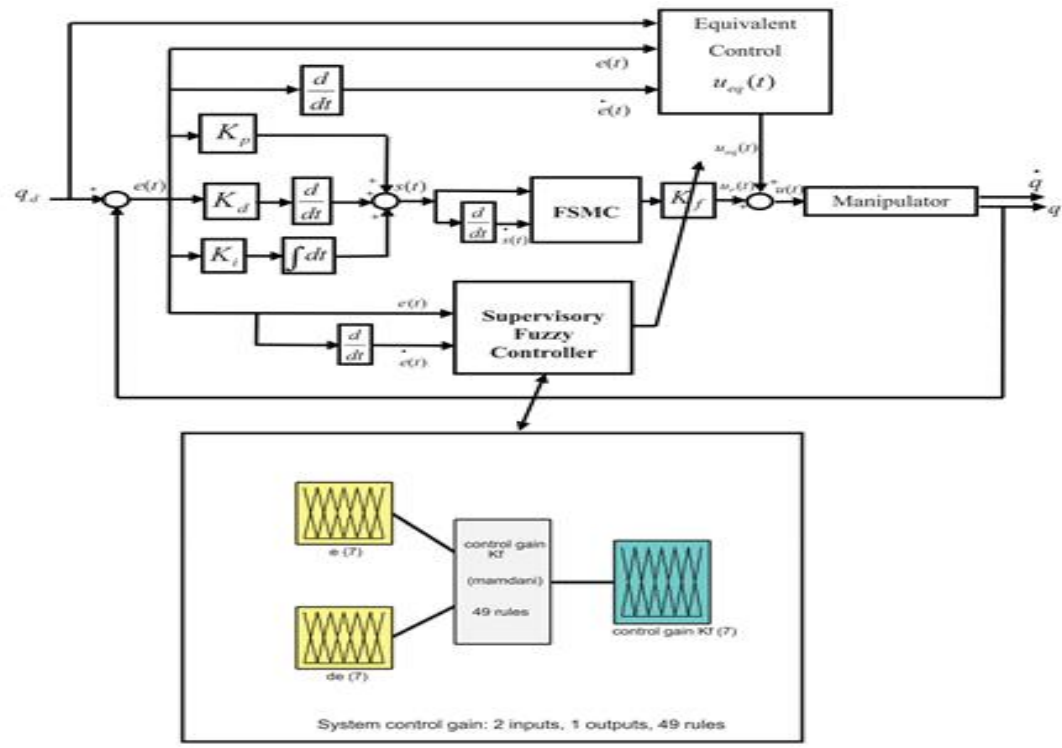

Fig7. Block diagram of Adaptive Fuzzy Sliding Mode Control with PID sliding surface

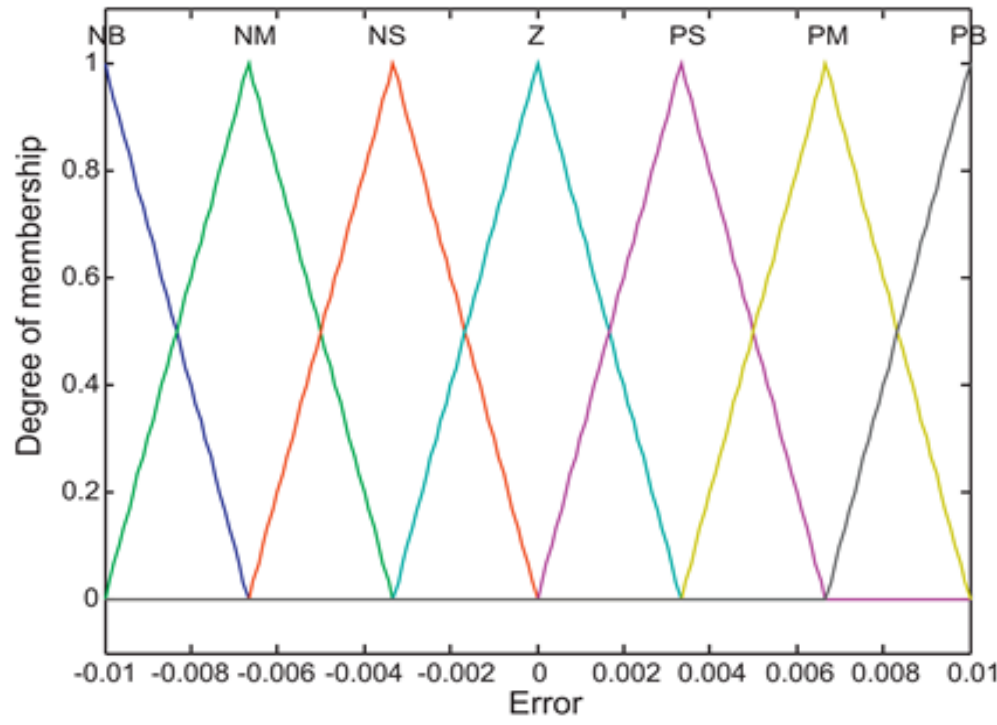

Fig8. Error Membership Function

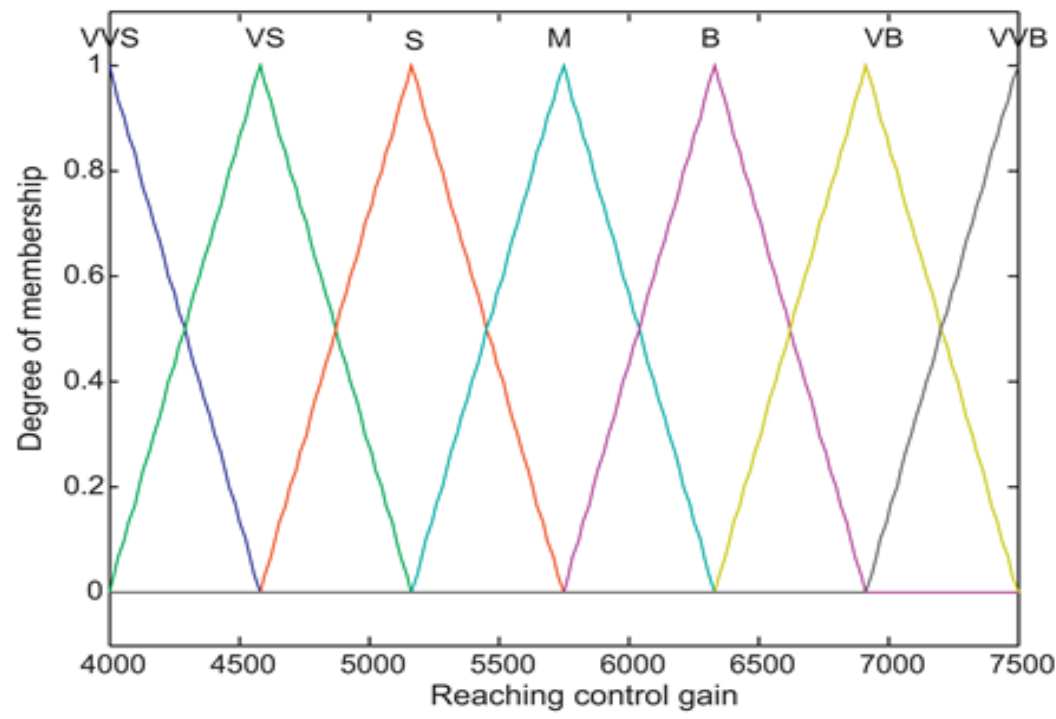

Fig9. $\mathbf{K}_{\mathrm{f}}$ Membership Function 


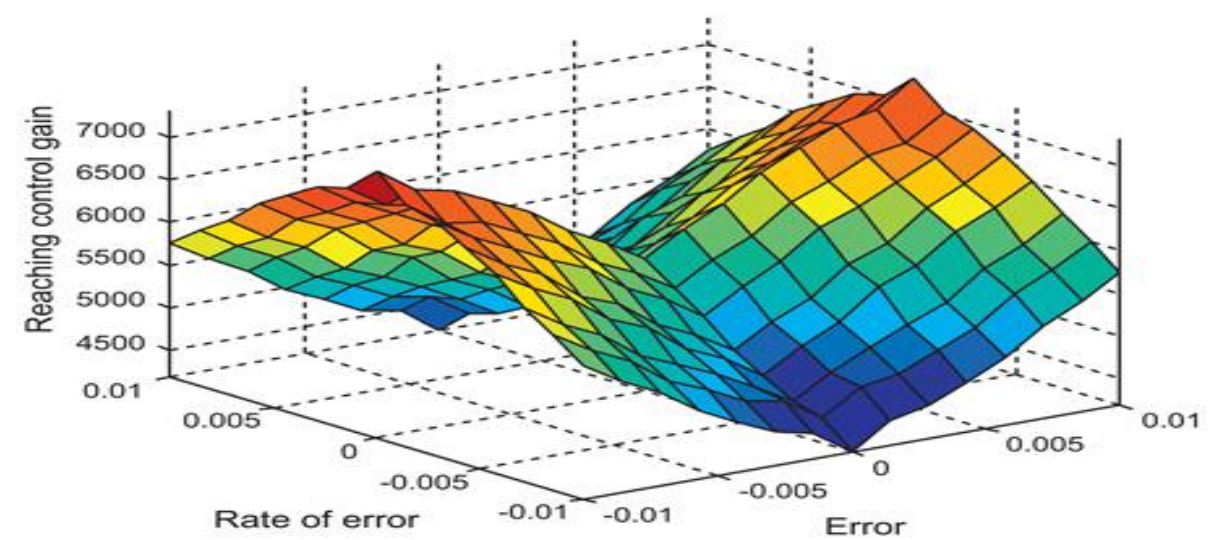

Fig10. $\mathbf{K}_{\mathrm{f}}$ fuzzy control surfaces

Table2. Matrix of adaptive fuzzy control rules

\begin{tabular}{llllllll}
\hline \multirow{2}{*}{$K_{f}$} & $e(t)$ & & & & & & \\
\cline { 2 - 8 }$\dot{e}(t)$ & $\mathrm{NB}$ & $\mathrm{NM}$ & $\mathrm{NS}$ & $\mathrm{Z}$ & $\mathrm{PS}$ & $\mathrm{PM}$ & $\mathrm{PB}$ \\
\hline NB & M & S & VS & VVS & VS & S & M \\
NM & B & M & S & VS & S & M & B \\
NS & VB & B & M & S & M & B & VB \\
Z & VVB & VB & B & M & B & VB & VVB \\
PS & VB & B & M & S & M & B & VB \\
PM & B & M & S & VS & S & M & B \\
PB & M & S & VS & VVS & VS & S & M \\
\hline
\end{tabular}

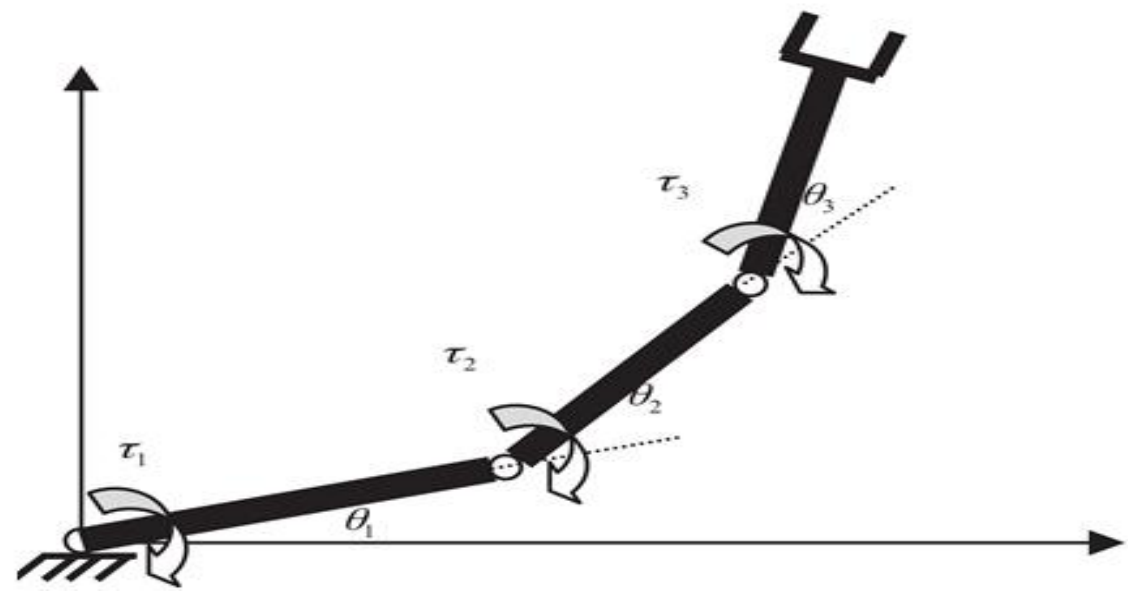

Fig11. Three linked Arm of Rigid Robot

\section{Simulation Results}

Suggested AFSMC for the Three linked Arm of Rigid Robot with 3 degree freedom (figure 11), has been tested. The movement equations and dynamic parameters of the Arm has been presented. The simulation was done by the Mathlab software. Uncertainties of equation 1 which show the dynamic effects are as below:

$$
\begin{aligned}
& F_{d} \dot{q}=\left[\begin{array}{l}
f_{d} \dot{q}_{1} \\
f_{d} \dot{q}_{2} \\
f_{d} \dot{q}_{3}
\end{array}\right]=\left[\begin{array}{l}
5 \dot{q}_{1} \\
5 \dot{q}_{2} \\
5 \dot{q}_{3}
\end{array}\right] \quad F_{s}(\dot{q})=\left[\begin{array}{l}
f_{s} \operatorname{sign}\left(\dot{q}_{1}\right) \\
f_{s} \operatorname{sign}\left(\dot{q}_{2}\right) \\
f_{s} \operatorname{sign}\left(\dot{q}_{3}\right)
\end{array}\right]=\left[\begin{array}{l}
5 \operatorname{sign}\left(\dot{q}_{1}\right) \\
5 \operatorname{sign}\left(\dot{q}_{2}\right) \\
5 \operatorname{sign}\left(\dot{q}_{3}\right)
\end{array}\right] \\
& \tau_{d}=\left[\begin{array}{l}
20+20 \sin (20(t-1))+30 \sin (10(t-0.5))+20 u(t-0.5)+20 u(t-1) \\
20+20 \sin (20(t-1))+30 \sin (10(t-0.5))+20 u(t-0.5)+20 u(t-1) \\
20+20 \sin (20(t-1))+30 \sin (10(t-0.5))+20 u(t-0.5)+20 u(t-1)
\end{array}\right]
\end{aligned}
$$

Revealing the effect of suggested method, two simulation has been performed. 


\subsection{High speed path detection control}

Used desirable path here has been given as: $q_{d}(t)=1-\cos (\pi t), 0 \leq t \leq 4$. The PID sliding surface parameters are: $K_{p}=\operatorname{diag}\{300,300,300\}, K_{i}=\operatorname{diag}\{250,250,250\}$ and $K_{d}=\operatorname{diag}\{20,20,20\}$. For ordinary SMC the impact control gain is set as: $K_{r}=\operatorname{diag}\{15000,15000,15000\}$. However it is set for FSMC as: $K_{r}=\operatorname{diag}\{5000,5000,5000\}$. For AFSMC the range of output gain, $K_{f}=\operatorname{diag}\left\{k_{f 1}, k_{f 2}, k_{f 3}\right\}$, is equal to $(4000,7500)$. Figures 12 to 14 show the 1,2 and 3 joint's path detection respectively. Figure 15 shows the detection error index of joints location for the 1, 2 and 3 joints. The IAE and ITAE indicators have been utilized for the comparison. The different error amounts for the various control strategies and diverse joints have been listed in table 3 .

\subsection{The picking up and inserting down duty}

The function of acceptable joint angle is chosen as: $q_{d}(t)=2+(-1+\tanh (10 \cos (0.25 t))), 0 \leq t \leq 12 \mathrm{sec}$. This function is widely used at industrial applications. The PID sliding surface are set as: $K_{p}=\operatorname{diag}\{20,20,20\}, K_{i}=\operatorname{diag}\{15,15,15\}$ and $K_{d}=\operatorname{diag}\{5,5,5\}$. For an ordinary SMC and FSMC the impact control gain is chosen as $K_{r}=\operatorname{diag}\{20000,20000,20000\}$ and $K_{f}=\operatorname{diag}\{5500,5500,5500\}$ respectively. For AFSMC the output gain range equals to $(4000,7500)$. The results are shown that suggested AFSMC is more applicable for all 3 joint. Table 1 and 2 approve this claim based on calculated efficiency indices. The IAE and ITAE for all 3 joints, reduced for AFSMC suggested method. Results revealed that suggested AFSMC has the more rapid detection and lower error than the SMC and FSMC methods.
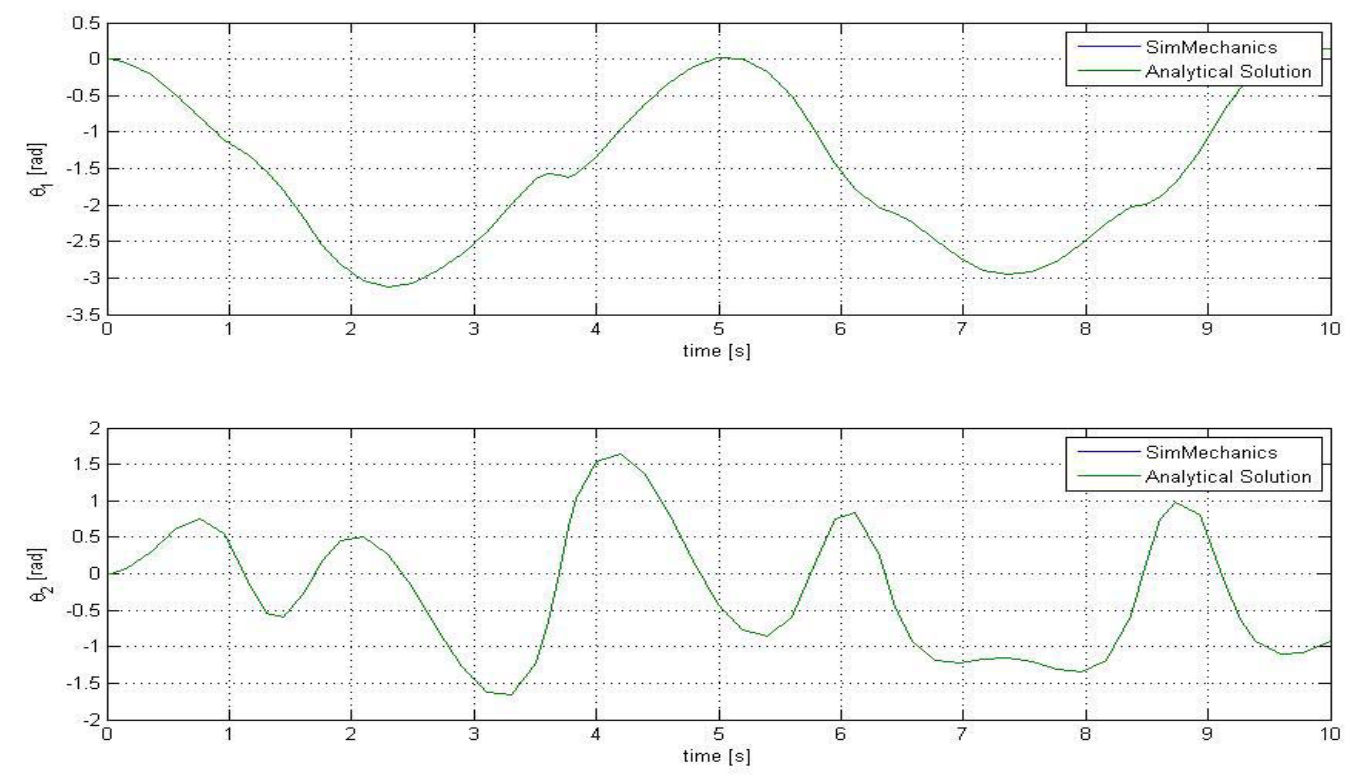

Fig12. Joint 1 path 

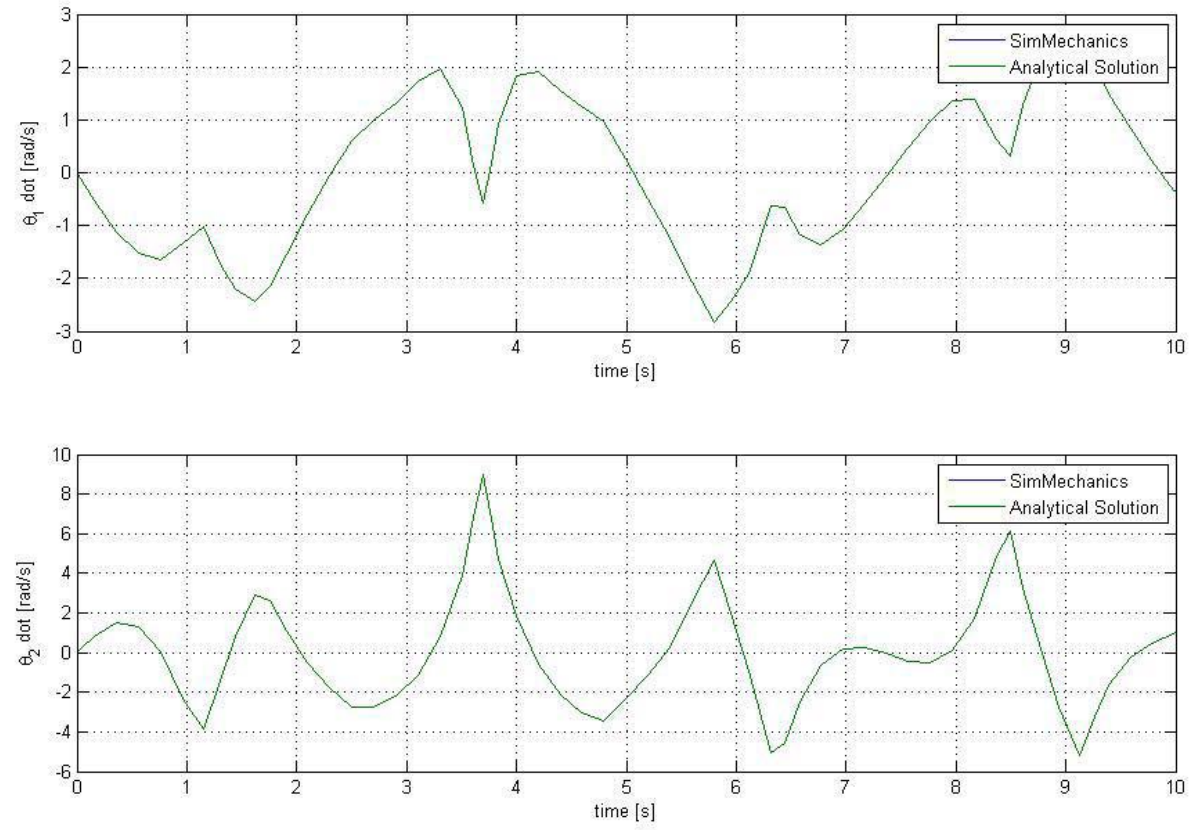

Fig13. Joint 2 path
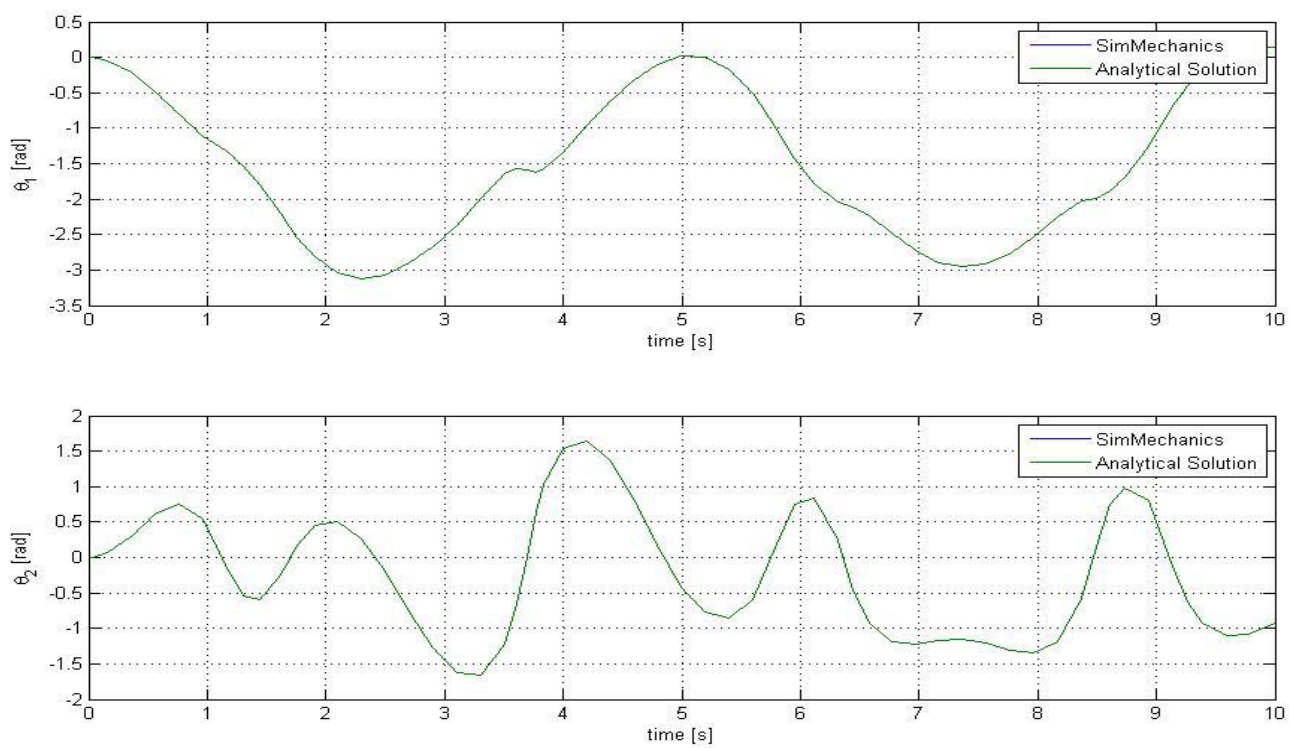

Fig14. Joint 3 path

Table3. The controller efficiency comparison in path detection control

\begin{tabular}{|c|c|c|c|c|c|c|}
\hline \multirow[t]{2}{*}{ Algorithm } & \multicolumn{3}{|l|}{ IAE } & \multicolumn{3}{|l|}{ ITAE } \\
\hline & Joint 1 & Joint2 & Joint3 & Joint 1 & Joint2 & Joint3 \\
\hline Conventional SMC & 3.92 & 3.62 & 5.01 & 16.81 & 11.41 & 17.4 \\
\hline FSMC & 0.54 & 0.54 & 0.54 & 2.51 & 2.52 & 2.52 \\
\hline AFSMC & 0.17 & 0.16 & 0.17 & 0.76 & 0.74 & 0.75 \\
\hline
\end{tabular}



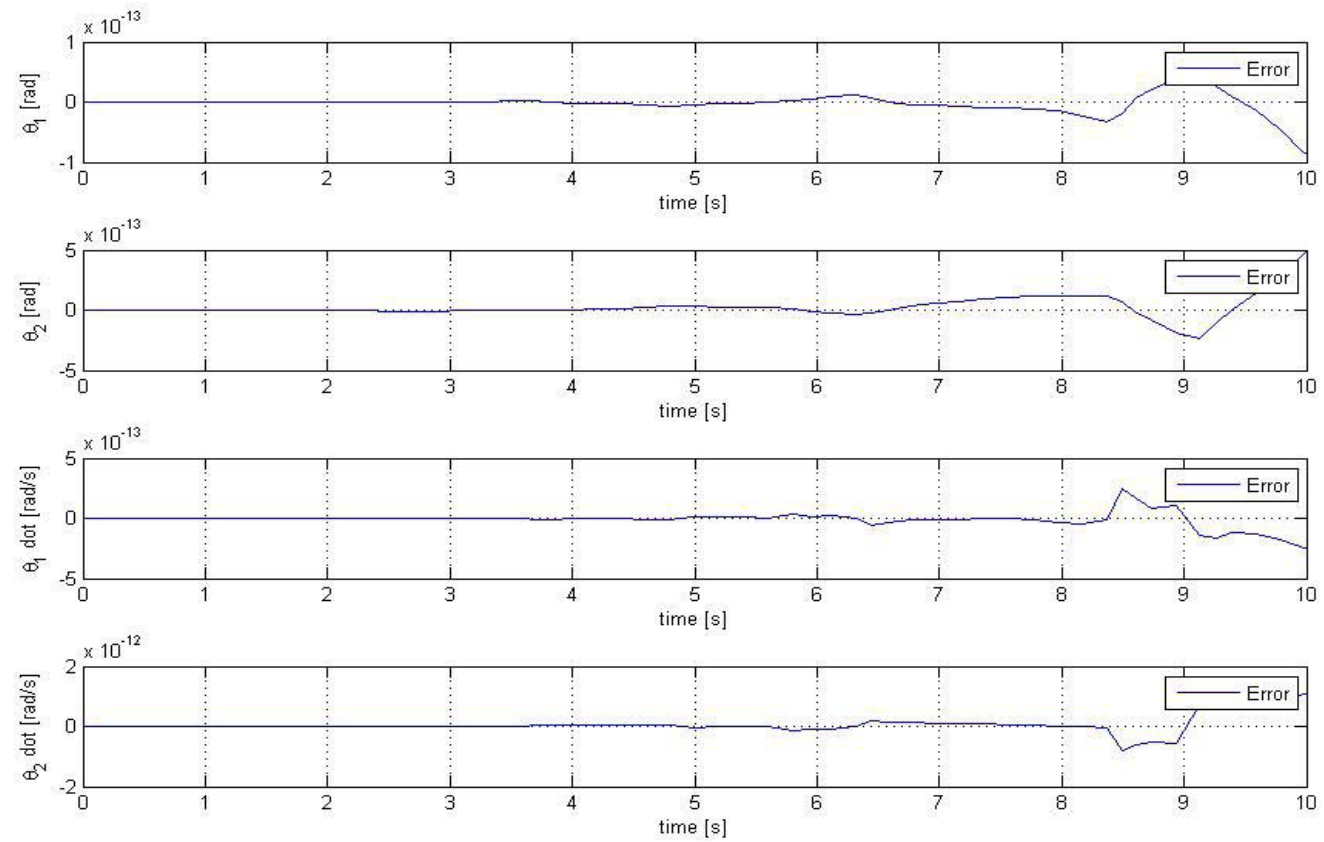

Fig15. The detection error of zero point in joint 1, 2 and 3

\section{Conclusion}

Present study explain the AFSMC method development for the successful robot arm control. Suggested AFSMC is stable and without the chattering has lots of industrial applications. This controller gives the stability from SMC method and chattering elimination of fuzzy logic.

Improving the FSMC efficiency, the impact control gain has been set online according to system error states by a supervised fuzzy controller. The suggested method doesn't need the uncertainty bounds and the system confusion. The current SMC methods are inapplicable in precise detection of rapid paths for the nonlinear or abundant nonlinear systems with huge uncertainty. The obtained efficiencies are not acceptable for the ordinary controllers; whereas it can be said that AFSMC has the more efficiency than the other methods in robot arm controlling. The results of simulation approved the suggested control method efficiency.

\section{References}

[1]. Ahmed F. Amera, Elsayed A. Sallamb, Wael M. Elawady, Department of Electrical and Control Engineering, Arab Academy for Science and Technology, Egypt, Tanta University, Egypt,(2011).

[2]. H. Yau, C. Chen, Chattering-free fuzzy sliding-mode control strategy for uncertain cha otic systems, Chaos Solitions Frast. 30 (2006) 709-718.

[3]. J.Soltani,MM.Rezaei, Robust control of an islanded multi- bus microgrid based on input-output feedback line arisation and sliding mode control , IET Generation Transmission \& Distribution - Institution of Electrical Engineers,(2015).

[4]. I. Eker, Sliding mode control with PID sliding surface and experimental application to an electromechanical plant, ISA Trans. 45 (January (1)) (2006) 109-118.

[5]. N.kherici, Y. Mohamed Ben ali, AliComputer Sciences Department, LRI, Badji Mokhtar-Annaba University, P.O. Box 12, 23000 Annaba, Algeriaa, Journal of Computational Science,(2015).

[6]. M.W. Spong, M. Vidyasagar, Robot Dynamics and Control, Wiley, New York,1989.

[7]. M. Zhu, Y. Li, Decentralized adaptive fuzzy sliding mode control for reconfigurable modular manipulators, Int. J. Nonlinear Control 20 (4) (2010) 472-488.

[8]. R.J. Wai, Fuzzy sliding-mode control using adaptive tuning technique, IEEE Trans. Ind. Electron. 54 (February (1)) (2007).

[9]. Y. Chen, J.L. Chang, Sliding-mode force control of manipulators, Proc. Natl. Sci. Counc. ROC(A) 23 (1999) 281-289.

[10]. L.M. Capisani, A. Ferrara, L. Magnani, Second order sliding mode motion control of rigid robot manipulators, in: Proceedings of the 46th IEEE Conference on Decision and Control ,New Orleans,LA, USA, (2007),PP.12-14.

[11]. H.F. Ho, Y.K. Wong, A.B. Rad, Robust fuzzy tracking control for robotic manipulators, simul, Modell.Pract. Theory 15 (2007) 801-816.

[12]. V.S. Vakula and K.R. Sudha, "Design of differential evolution algorithm-based robust fuzzy logic power system stabiliser using minimum rule base", Generation, Transmission \& Distribution IET, Vol. 6, Iss. 2,( 2012), pp. 121-132. 